\title{
Impaired White Matter Integrity and Social Cognition in High-Function Autism: Diffusion Tensor Imaging Study
}

\author{
Woo Young $\mathrm{Im}^{1,2}$, Ji Hye Ha ${ }^{3}$, Eun Joo Kim ${ }^{4,5}$, Keun-Ah Cheon ${ }^{4,5}$, Jaeil Cho ${ }^{6}$, and Dong-Ho Song ${ }^{4,5} \bowtie$ \\ ${ }^{1}$ Department of Psychiatry and Myunggok Medical Research Institute, Konyang University College of Medicine, Daejeon, Republic of Korea \\ ${ }^{2}$ Department of Medicine, the Graduate School of Yonsei University, Seoul, Republic of Korea \\ ${ }^{3}$ Department of Psychiatry, Seoul Metropolitan Eunpyeong Hospital, Seoul, Republic of Korea \\ ${ }^{4}$ Department of Psychiatry, Yonsei University College of Medicine, Seoul, Republic of Korea \\ ${ }^{5}$ Institute of Behavioral Science in Medicine, Yonsei University College of Medicine, Seoul, Republic of Korea \\ ${ }^{6}$ Yonsei Soul Psychiatric Clinic, Seoul, Republic of Korea
}

Objective It is known that many of the cognitive and social deficits associated with autism can arise from abnormal functional connectivity between brain networks. This aberrant functional connectivity in autism spectrum disorders (ASD) can be explained by impaired integrity of white matter tracts that link distant regions of the networks.

Methods We investigated white matter in 9 children with high-function autism (HFA) compared to 13 typically developing controls using diffusion tensor imaging (DTI). The aim of this research is to provide supporting evidence for abnormalities in neural connectivity as an underlying pathophysiology of the main characteristics of ASD.

Results We found impairment of neural connectivity, mainly in association fiber tracts as evidenced by decreased fractional anisotropy (FA), the index of white matter integrity, of these tracts. Among them, inferior fronto-occipital fasciculus (IFOF) had a significant relationship with ADI-R score. The inferior longitudinal fasciculus (ILF) and superior longitudinal fasciculus (SLF) also showed decreased FA. Decreased FA of ILF and SLF had negative correlations with scores of social interaction.

Conclusion These findings suggest that widespread abnormalities in association fiber tracts may contribute to both core and associated symptoms of ASD.

Psychiatry Investig 2018;15(3):292-299

Key Words High-function autism, Diffusion tensor imaging, Brain network, Functional connectivity, White matter.

\section{INTRODUCTION}

Autism spectrum disorder (ASD) is a neurodevelopmental disorder that has persistent deficits in social communication and social interaction and shows restricted and repetitive patterns of behavior, interests, or activities. ${ }^{1}$ Despite enormous research efforts the etiology of autism is unknown; however, several studies have showed strong evidence suggesting that many of the cognitive and social deficits associated with autism arise from abnormal functional connectivity between

Received: November 22, 2016 Revised: June 3, 2017

Accepted: August 15, 2017

$\triangle$ Correspondence: Dong-Ho Song, $\mathrm{MD}, \mathrm{PhD}$

Department of Psychiatry and Institute of Behavioral Science in Medicine, Yonsei University College of Medicine, 50-1 Yonsei-ro, Seodaemun-gu, Seoul 03722, Republic of Korea

Tel: +82-2-2228-1626, Fax: +82-2-313-0891, E-mail: dhsong@yuhs.ac

(a) This is an Open Access article distributed under the terms of the Creative Commons Attribution Non-Commercial License (http://creativecommons.org/licenses/bync/4.0) which permits unrestricted non-commercial use, distribution, and reproduction in any medium, provided the original work is properly cited. brain networks. ${ }^{2-5}$ This aberrant functional connectivity in ASD can be explained by impaired integrity of white matter tracts that link distant regions of the networks. White matter is a complex of myelinated bundles of axons that enable electrical signals to be transmitted between distant brain regions. To perform complex socioemotional and communication tasks, efficient conduction of information among cortico-cortical white matter tracts is necessary. ${ }^{6}$

Diffusion tensor imaging (DTI) is one of the most useful methods to assess the microstructure of neural fiber tracts without any intrusive intervention. DTI measures diffusion properties, such as the magnitude, anisotropy (variation of diffusion properties with direction), and orientation of anisotropic diffusion of water within tissues. ${ }^{7}$ The most commonly used parameter of diffusion anisotropy is fractional anisotropy (FA). FA is sensitive to developmental changes and pathological differences in axonal density, size, myelination, and the coherence of organization of fibers within a voxel; therefore, 
it can represent an index of the structural integrity of white matter. Several studies reported evidence of reductions in fractional anisotropy (FA) in ASD. Barnea-Goraly et al. ${ }^{8}$ performed the first DTI study of ASD. They found that seven children with autism (mean age 14.6, IQ>70) had reduced FA compared with normal controls in several brain regions including ventromedial prefrontal cortices, anterior cingulate cortex, temporal lobes, and corpus callosum. Lee et al. ${ }^{9}$ reported decreased FA of superior, middle, and inferior temporal white matter in patients with autistic disorder aged 7-33 years. Alexander et al. ${ }^{10}$ revealed that the corpus callosum, an essential structure for interhemispheric communication, has lower FA and higher radial diffusivity in autistic individuals aged 7-33. Cheung et al..$^{11}$ investigated FA and its correlation with the diagnostic phenotype described by scores on the Autism Diagnostic Interview-Revised (ADI-R) in children with autism (age 6-14 years, IQ $>70$ ). They found that FA in autistic children was significantly lower in bilateral prefrontal and temporal regions than that of normal controls. Importantly, they also found a meaningful correlation between a lower FA of fronto-striato-temporal pathways and higher ADI-R diagnostic scores in children with ASD showing aberrancy of white matter structure involved in social cognition.

All the studies mentioned above used voxel-based morphometry (VBM) style analyses, which were traditionally used to localize differences in grey matter density. Though they showed consistent results of reduced FA in various brain areas of subjects with ASD, these methods are limited by misalignment and arbitrarily applied smoothing parameters that can induce abnormal results. ${ }^{12,13}$ Recently, a newer approach known as tract based spatial statistics (TBSS) has been used to resolve methodological issues associated with VBM style analyses. TBSS is a voxel-based approach to DTI that allows determination of significant differences in FA images of multiple subjects between study groups. ${ }^{12}$ Because FA map does not have anatomical information, we use TBSS instead of VBM, which was applied in gray matter. The TBSS, combined with a VBA were applied in Jou et al. ${ }^{14}$ to a small group of older ASD and control children. The FA was reduced, especially, in the forceps minor, inferior fronto-occipital fasciculus, and superior longitudinal fasciculus. Regional distributions of differences between young ASD and control children were examined in Walker et al. ${ }^{15}$ using the TBSS and the whole-brain VBA. While the FA values were reduced in various brain regions.

In the present study, we investigated white matter in children and adolescents with high-function autism (IQ $>80)$ compared to normal controls using DTI. The first aim of the study was to identify differences in white matter in children and adolescents with high-function autism compared with a normal control group using FA as a DTI index of underlying white matter mi- crostructure to prove aberrancy of brain networks in autism spectrum disorder. The second aim was to examine whether social cognition and communication measured by ADI-R has any relationship with FA of meaningful areas in TBSS analyses to identify correlations between abnormalities of brain connectivity and social cognition. We hoped to provide evidence that abnormalities in neural connectivity are an underlying pathophysiology of the main characteristics of autism spectrum disorder.

\section{METHODS}

\section{Participants}

Participants were 22 children and adolescents with fullscale IQ $>80$ as estimated by the Wechsler Intelligence Scale for Children-IV. ${ }^{16}$ Children and adolescents with a history of comorbid psychiatric or medical conditions (e.g., epilepsy), a history of head injury, or a genetic disorder associated with autism (e.g., fragile $\mathrm{X}$ syndrome) were excluded.

High-functioning autism (HFA) is a term applied to people with autism who are deemed to be cognitively "higher functioning" (with an IQ of 70 or greater) than other people with autism. The HFA group consisted of nine participants aged 9-14 years (8 males and 1 female), who were recruited through the Severance Children's Hospital at Yonsei School of Medicine (Seoul, Korea). The autism patients and parents who visited the hospital were consented to the agreement. Diagnoses of autism spectrum disorders were based on DSM- 5 criteria and supported by the Autism Diagnostic Observation Schedule $(\mathrm{ADOS})^{17}$ and Autism Diagnostic Interview-Revised (ADIR). ${ }^{18}$ All nine participants were diagnosed as autism spectrum disorder. Biological, IQ and diagnostic scores of children and adolescents with HFA are represented in Table 1.

The control group consisted of 13 typically developing children and adolescents aged 7-14 years (all males). The control group was recruited through the recruitment advertisement in the hospital. As shown in Table 2, demographic data are represented and there are no significant differences in age and verbal, performance, and functional IQ scores between HFA and normal control groups. All participants were right handed.

The study was approved by the Institutional Review Board of the Yonsei University College of Medicine and informed consent was obtained for all participants (IRB No. 3-20080072).

\section{Data acquisition}

All brain scans were performed using a Philips 3T scanner (Intera Achieva, Philips Medical System, Best, The Netherlands) with a SENSE head coil. Diffusion tensor images were obtained using a single-shot echo-planar acquisition with the 
Table 1. Biological, IQ and diagnostic scores of children and adolescents with HFA

\begin{tabular}{|c|c|c|c|c|c|c|c|c|c|c|c|}
\hline \multirow{2}{*}{ Participant } & \multicolumn{3}{|c|}{ Biological } & \multicolumn{3}{|c|}{ IQ } & \multicolumn{2}{|c|}{ ADOS } & \multicolumn{3}{|c|}{ ADI-R } \\
\hline & Age & Sex & Handedness & Verbal & Performance & Functional & $\mathrm{COM}$ & SOC & SOC & $\mathrm{COM}$ & STB \\
\hline 1 & 13 & M & Rt & 91 & 100 & 94 & 7 & 13 & 25 & 21 & 4 \\
\hline 2 & 10 & M & Rt & 104 & 102 & 104 & 6 & 12 & 20 & 16 & 5 \\
\hline 3 & 13 & M & Rt & 123 & 116 & 122 & 3 & 5 & 7 & 8 & 3 \\
\hline 4 & 14 & M & Rt & 127 & 116 & 124 & 3 & 4 & 19 & 17 & 9 \\
\hline 5 & 12 & $\mathrm{~F}$ & Rt & 104 & 102 & 104 & 5 & 8 & 19 & 16 & 6 \\
\hline 6 & 10 & M & Rt & 88 & 105 & 94 & 3 & 7 & 18 & 20 & 9 \\
\hline 7 & 9 & M & Rt & 101 & 66 & 82 & 5 & 6 & 26 & 20 & 6 \\
\hline 8 & 12 & M & Rt & 125 & 116 & 123 & 3 & 6 & 13 & 7 & 6 \\
\hline 9 & 11 & M & Rt & 95 & 108 & 101 & 4 & 10 & 20 & 17 & 8 \\
\hline
\end{tabular}

ADOS (COM: Autism cut-off 4, Autism spectrum cut-off 2), ADOS (SOC: Autism cut-off 7, Autism spectrum cut-off 4), ADI-R (SOC: Autism spectrum cut-off 10), ADI-R (COM: Autism spectrum cut-off 8), ADI-R (STB: Autism spectrum cut-off 3). HFA: high-function autism, IQ: intelligence quotient, ADOS: autism diagnostic observation schedule, ADI-R: autism diagnosis interview-revised, COM: communication, SOC: social interaction, STB: restricted, repetitive, stereotyped behaviors

Table 2. Demographic data for the HFA and control groups

\begin{tabular}{lccc}
\hline & HFA $(\mathrm{N}=9)$ & Control $(\mathrm{N}=13)$ & p value \\
\hline Age & $11.6 \pm 1.7$ & $11.5 \pm 2.1$ & 0.909 \\
FS-IQ & $105.3 \pm 14.8$ & $114.2 \pm 11.7$ & 0.154 \\
V-IQ & $106.4 \pm 15.0$ & $118.3 \pm 12.5$ & 0.68 \\
P-IQ & $103.4 \pm 15.5$ & $105.6 \pm 9.5$ & 0.714 \\
\hline
\end{tabular}

HFA: high-function autism, FS-IQ: functional intelligence quotient, V-IQ: verbal intelligence quotient, P-IQ: performance intelligence quotient, control: typically developing children

following parameters: $112 \times 112$ acquisition and a $128 \times 128$ reconstructed matrix, field of view $=220 \mathrm{~mm}$, voxel size $=1.72 \times$ $1.72 \times 2 \mathrm{~mm}^{3}$, SENSE factor 2, echo time=70 ms; shortest repetition time $=13,000 \mathrm{~ms}$, flip angle $=90^{\circ}$, slice gap $=0 \mathrm{~mm}$, two averages per slice, $b$-factor $=600 \mathrm{~s} / \mathrm{mm}^{2}$, non-cardiac gating and 70 axial slices. Diffusion-weighted images were gathered from 45 non-collinear, non-coplanar directions with a baseline image without diffusion weighting. The spatial distortions induced by eddy currents and motion artifacts in diffusionweighted images were corrected by registering the diffusionweighted images to the non-diffusion-weighted b0 image using a low order non-linear transformation. ${ }^{19}$

\section{TBSS analysis}

Preprocessing for DTI analysis was performed using FMRIB Software Library (FSL; Oxford, UK; http://www.fmrib. ox.ac.uk/fsl). A diffusion tensor model was arranged for each voxel with the generation of FA and mode images using FMRIB's diffusion toolbox in FSL. The TBSS tool in FSL was used to analyze tract-based differences in FA values between ASD subjects and healthy control subjects. ${ }^{12}$

All subjects' FA images were aligned into $1 \times 1 \times 1 \mathrm{~mm}^{3} \mathrm{MNI} 152$ (Montreal Neurological Institute) standard space using the nonlinear registration and a mean FA image was generated from the transformed FA images. A mean skeleton image was created from the mean FA image. Each subject's aligned FA image was projected onto the mean FA skeleton. The threshold for the mean FA skeleton we set was 0.2.

\section{Statistical analysis}

Statistical analyses of the FA values were performed with a tract-based spatial statistics technique using the FMRIB Diffusion Toolbox implemented in the FMRIB software library (http://www.fmrib.ox.ac.uk/fsl/tbss/index.html). ${ }^{12,20,21}$ Group differences in FA between the patient and control group were evaluated with a non-parametric 1000 permutation-based randomized test ${ }^{19}$ and inference using threshold-free cluster enhancement(TFCE) ${ }^{22}$ implemented in the FMRIB software library to correct $(p<0.05)$ for multiple comparisons. If there are not any significant difference, we explored the group difference or relationship of the FA value with clinical variable with threshold of TFCE uncorrected $\mathrm{p}<0.001$ regarding to small sample size in this study. In order to examine the relationships between WM integrity and behavior, we calculated Pearson correlations between FA values and scores in the ADOS and ADI-R score in HFA. Age and gender was as covariates. The JHU White-Matter Tractography Atlas was used to identify the white matter pathways involved. ${ }^{23}$

\section{RESULTS}

As shown in Table 3, there were regions with significant reduced fractional anisotropy (FA) in subjects with HFA vs. Controls.

As shown in Table 4, there were regions in which FA is negatively correlated with ADI-R scores in HFA. 
Results of DTI are shown in Figures 1-5. The threshold was set to uncorrected $\mathrm{p}<0.001$ for display and the results were displayed using the tbss_fill command. The same criteria as the threshold for the reported results were applied. Tract-based spatial statistics analysis revealed significantly lower FA in left inferior fronto-occipital fasciculus (IFOF), left superior longitudinal fasciculus (SLF), left inferior longitudinal fasciculus (ILF), and right amygdala (AMY) in the HFA group compared to the normal control group when sex and age are regressed out.

Table 3. Regions with significant reduced FA in HFA vs. Controls

\begin{tabular}{|c|c|c|c|c|c|c|}
\hline \multirow{2}{*}{ Regions } & \multicolumn{3}{|c|}{ MNI coordinates } & \multirow{2}{*}{ HFA $(\mathrm{N}=9)$} & \multicolumn{2}{|c|}{ FA (median) } \\
\hline & $\mathrm{x}$ & $\mathrm{y}$ & $\mathrm{z}$ & & Control $(\mathrm{N}=13)$ & $\mathrm{p}$ \\
\hline Inferior fronto-occipital fasciculus L & -38 & -56 & 3 & 0.232 & 0.306 & $0.000^{* *}$ \\
\hline Superior longitudinal fasciculus L & -48 & -2 & 41 & 0.303 & 0.372 & $0.000^{* *}$ \\
\hline Inferior longitudinal fasciculus $\mathrm{L}$ & -40 & -56 & 3 & 0.501 & 0.589 & $0.000^{* *}$ \\
\hline Amygdala R & 19 & 0 & -25 & 0.202 & 0.254 & $0.000^{* *}$ \\
\hline
\end{tabular}

${ }^{* *} \mathrm{p}<0.05$. HFA: high-function autism, MNI: montreal neurologic institute, FA: fractional anisotropy

Table 4. Regions in which fractional anisotropy (FA) is negatively correlated with ADI-R scores in HFA

\begin{tabular}{llllrrr}
\hline \multirow{2}{*}{ Behavioral score } & Correlation & & & \multicolumn{2}{c}{ MNI coordinates } \\
\cline { 5 - 7 } & & & & $\mathrm{x}$ & $\mathrm{y}$ \\
\hline ADI-R (SOC) & Negative & $\mathrm{L}$ & Corticospinal tract & -10 & -21 & -23 \\
& Negative & $\mathrm{L}$ & Uncinate fasciculus & -32 & 6 & -8 \\
& Negative & $\mathrm{L}$ & Inferior fronto-occipital fasciculus & -40 & -20 & -8 \\
& Negative & $\mathrm{L}$ & Inferior longitudinal fasciculus & -28 & -77 & -25 \\
& Negative & $\mathrm{L}$ & Superior longitudinal fasciculus & -37 & 28 \\
\hline
\end{tabular}

HFA: high-function autism, ADI-R: autism diagnosis interview-revised, SOC: social interaction
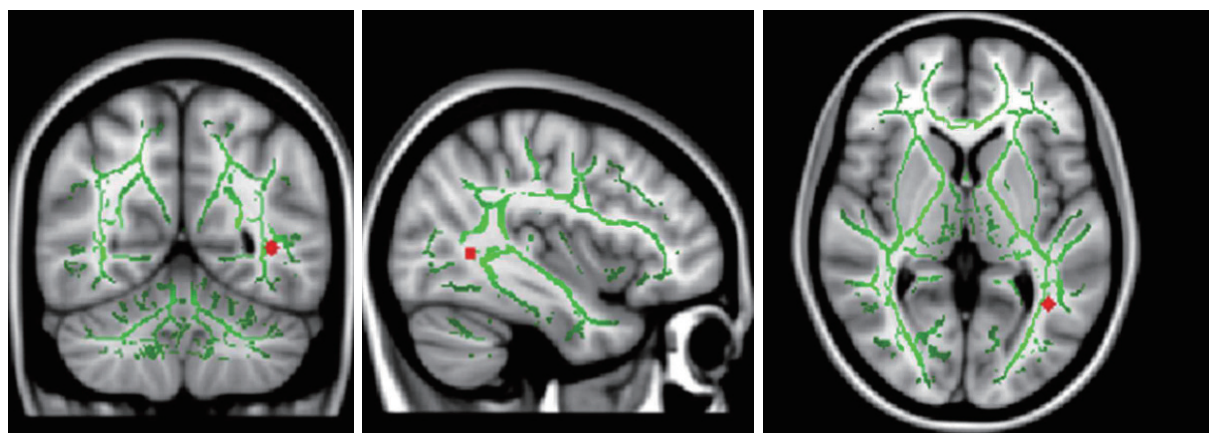

Figure 1. TBSS analysis demonstrated decreased FA (red) in left inferior fronto-occipital fasciculus (IFOF) in children and adolescents with high-function autism compared with normal controls. Left IFOF: MNI (x, y, z)=-38, -56, 3. TBSS: tract based spatial statistics.
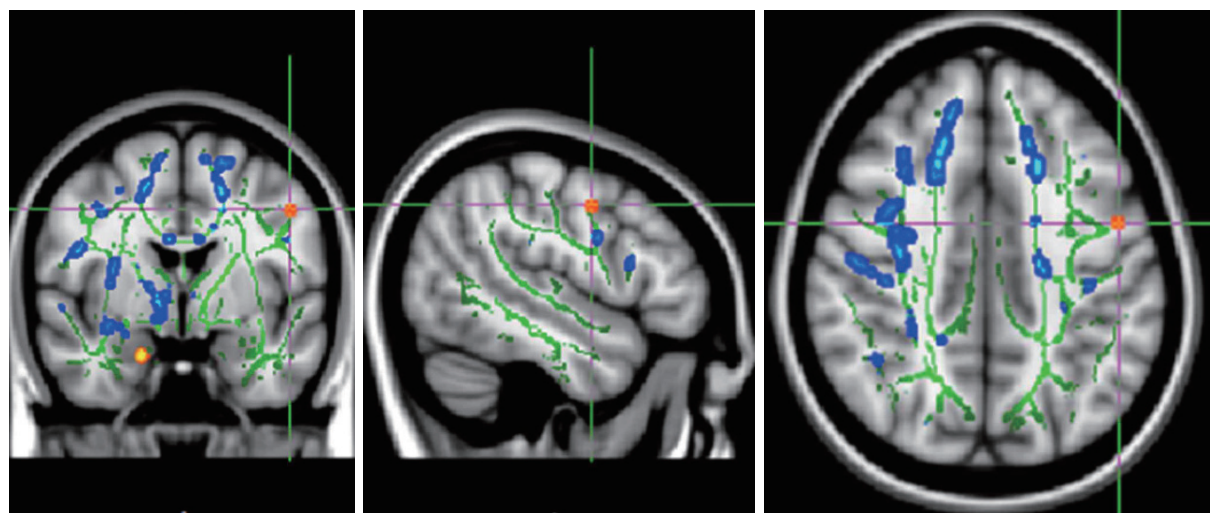

Figure 2. TBSS analysis demonstrated decreased FA (red) in left superior longitudinal fasciculus (SLF) in children and adolescents with high-function autism compared with normal controls. Left SLF: MNI $(x, y, z)=-48,-2,41$. TBSS: tract based spatial statistics. 

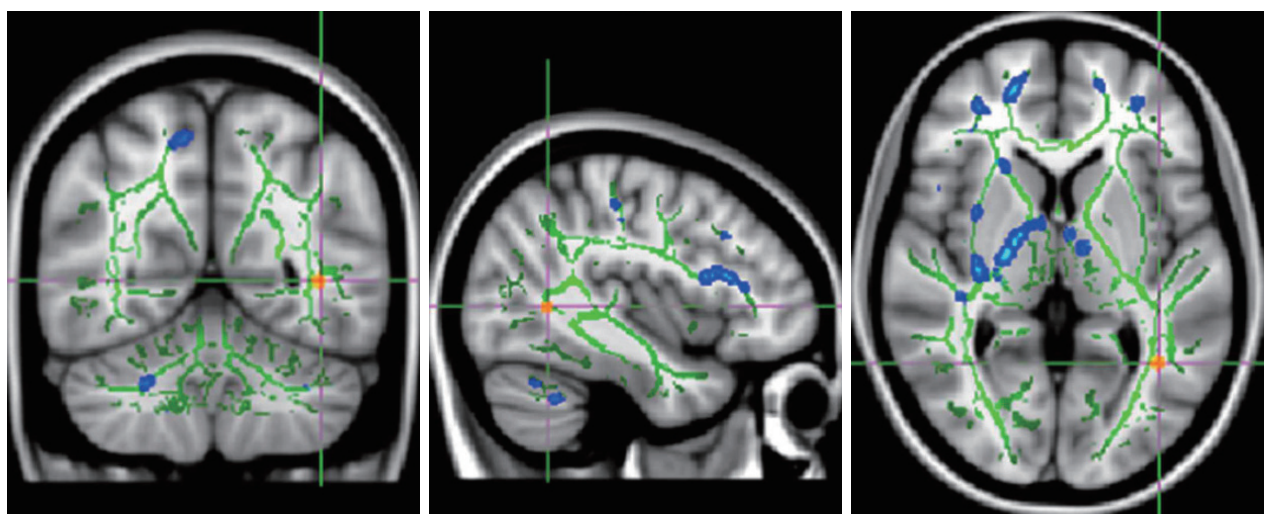

Figure 3. TBSS analysis demonstrated decreased FA (red) in left inferior longitudinal fasciculus (ILF) in children and adolescents with highfunction autism compared with normal controls. Left ILF: MNI $(x, y, z)=-40,-56,3$. TBSS: tract based spatial statistics.
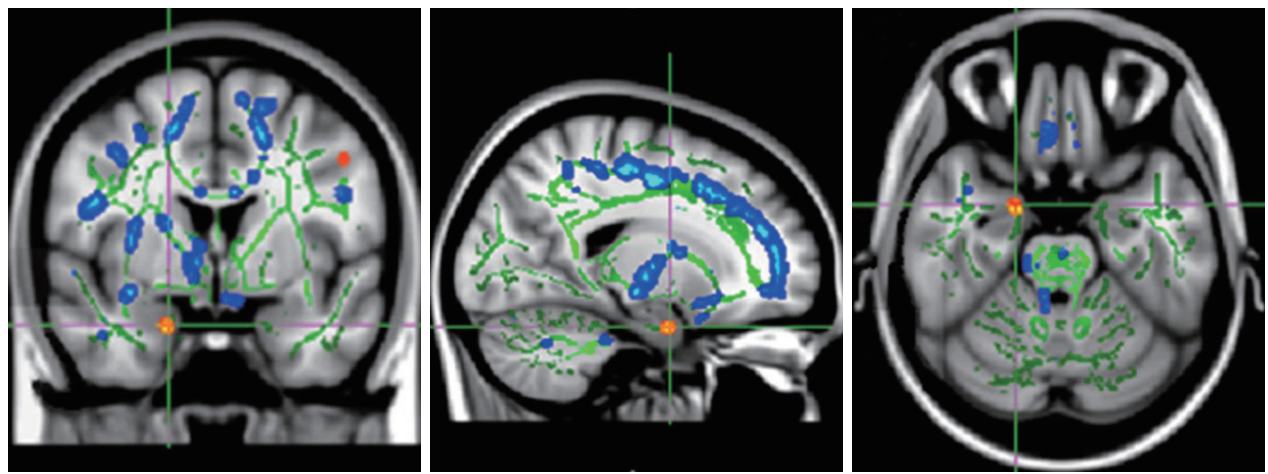

Figure 4. TBSS analysis demonstrated decreased FA (red) in right amygdala in children and adolescents with high-function autism compared with normal controls. Right amygdala: $\mathrm{MNI}(x, y, z)=19,0,-25$. TBSS: tract based spatial statistics.

There were significant negative correlations between social interaction scores of ADI-R and FA of left corticospinal tract, left uncinate fasciculus, left inferior fronto-occipital fasciculus, left inferior longitudinal fasciculus, and left superior longitudinal fasciculus (Figure 5).

\section{DISCUSSION}

In the present study we compared the white matter structure of individuals with high-function autism and normal controls using TBSS. We found significantly decreased FA in left IFOF, left SLF, left IFL, and right AMY in the HFA group.

The finding of reduced FA in the current study is consistent with the majority of DTI studies performed in ASDs which also report mainly reductions in FA. It is important to note that despite the between-study differences in subject characteristics, image acquisition, and processing/analysis methodology, FA is consistently reduced, although the distribution of the reduction is variable.

In Perkins's study, ${ }^{24}$ there is evidence emerging from Diffusion Tensor Imaging (DTI) research that autism spectrum disorders (ASD) are associated with greater impairment in the left hemisphere. In the left hemisphere, participants with
HFA demonstrated significantly reduced FA in predominantly thalamic and fronto-parietal pathways. In this study, we also showed decreased FA findings in left hemispheres such as left IFOF, left SLF, and left IFL.

In Bakhtiari et al., ${ }^{25}$ Adolescents with ASD showed decreased FA values bilaterally over a very large region compared to NT. Decreased FA values were found bilaterally in the inferior fronto-occipital fasciculus (IFOF), the inferior longitudinal fasciculus (ILF), the superior longitudinal fasciculus (SLF), the uncinate fasciculus (UNC), the anterior thalamic radiation (ATR), the cingulum in its cingulate section, the corticospinal tract (CT) and in the corpus callosum (CC), as well as in the right forceps major and minor, and in the right hippocampus section of the cingulum. The fibers in which the TBSS revealed FA negatively correlated with Autism-spectrum Quotient (AQ) were similar to those where previous TBSS was found with reduced FA in ASD compared with controls. ${ }^{26-28}$ The locations of clusters were similar to those of previous studies reporting IFOF/ILF, CC, AF and ATR.

In Catanis Study, ${ }^{28}$ Decreased FA was also found in the IFOF, connecting the occipital cortex through the uncinate fasciculus, and terminating in the orbitofrontal cortex and a negative correlation between FA in IFOF was found with ADOS and 
ADI-R communication scores in ASD. Considering its spatial connection, IFOF may play a crucial role in the neurobiology of ASD. It has direct connections to the fusiform gyrus, which is responsible for facial perception, and provides connections between all major lobes of the human brain: frontal, temporal, parietal, and occipital. ${ }^{29}$ This broad neuroanatomic connection is a unique quality of the IFOF and corresponds to the broad spatial distribution of brain structures involved in social cognition including the fusiform gyrus (temporal lobe), amygdala (temporal lobe), superior temporal sulcus (temporal lobe), ventromedial prefrontal cortex (frontal lobe), temporoparietal junction (temporal and parietal lobes), and somatosensory cortices (parietal lobe). The IFOF is important in higher-level visual processing such as control of action and
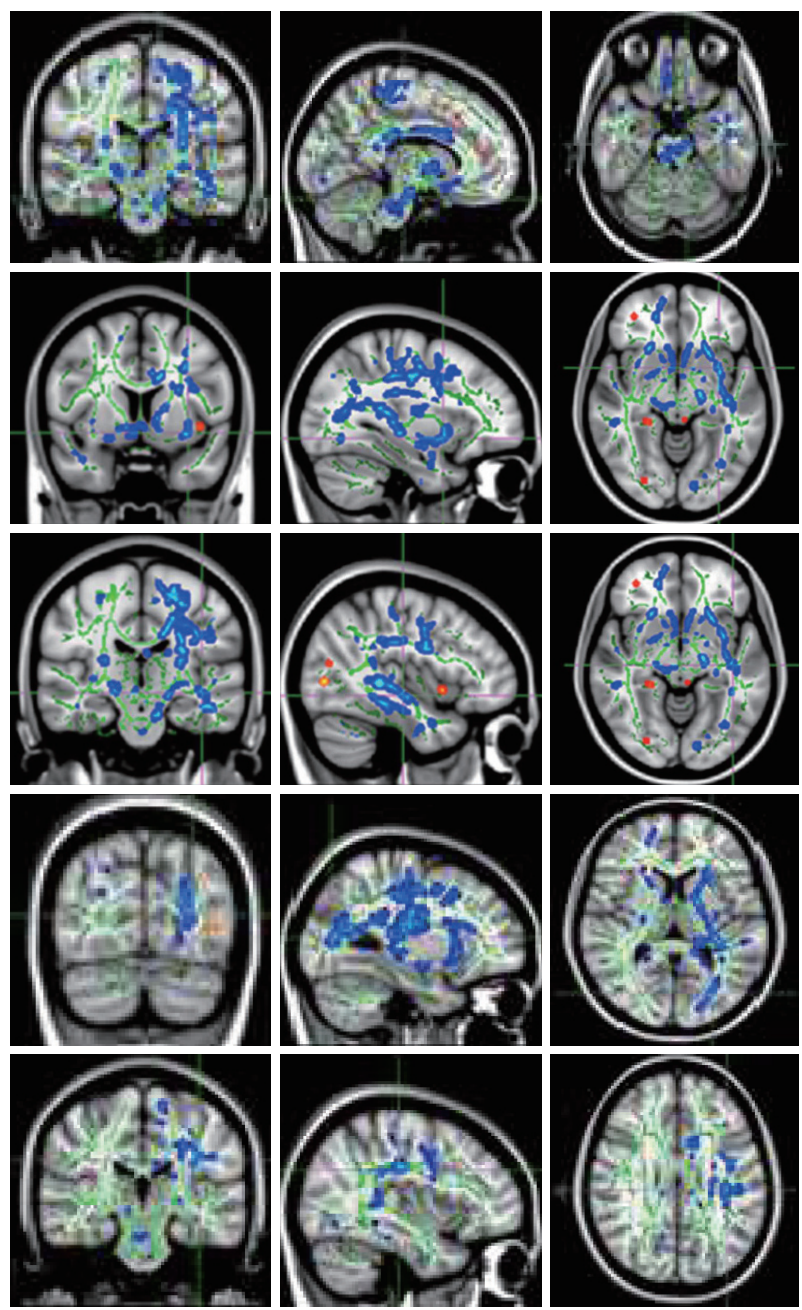

Figure 5. Social communication scores of $A D I-R$ have negative correlations (blue areas) with FA of left corticospinal tract [MNI ( $\mathrm{x}$, $y, z)=-10,-21,-23]$, left uncinate fasciculus [MNI $(x, y, z)=-32,6$, -8], left inferior fronto-occipital fasciculus [MNI $(x, y, z)=-40,-20$, -8], left inferior longitudinal fasciculus [MNI $(x, y, z)=-28,-77,9]$, and left superior longitudinal fasciculus [MNI $(x, y, z)=-37,-25,28]$. ADI-R: Autism Diagnostic Interview-Revised, FA: fractional anisotropy. recognition of faces. ${ }^{30,31}$ Other DTI studies ${ }^{32,33}$ have also shown IFOF repeatedly suggesting that it was peculiar to human beings in Thiebaut de Schotten et al. ${ }^{34}$ Furthermore, a Philippi's study of brain injury indicated that IFOF was associated with perception of facial expression, ${ }^{35}$ which has been reported to be impaired in people with ASD. ${ }^{36}$ In Losh et al. ${ }^{37}$ and Weng et $\mathrm{al}^{38}$ the processing of facial expression is aberrant in people with ASD. Thus, variation of fiber integrity of IFOF might lead to autistic features, as the fiber plays a key role in processing social information. In the present study, left IFOF had significantly lower FA in the HFA group than in the normal control group and FA of left IFOF had negative correlations with social interaction scores of ADI-R. These findings suggest that abnormal connections between brain structures can be a potential mechanism for known impairments in social interaction in ASD.

The next tract of interest in this study is the ILF, which also can be impaired in ASD. The ILF, extending from the occipital cortex into superior, middle and anterior temporal lobes, mediates connectivity between the superior temporal sulcus, which processes biological motion and eye gaze, ${ }^{39}$ the fusiform face area, and the amygdala, which is involved in processing the social significance of facial expressions. ${ }^{40}$ Some research shows that children with ASD have a facial emotion recognition (FER) deficit. ${ }^{41,42}$ Many children with ASD have difficulty identifying facial emotions. ${ }^{43}$ Individuals with ASD have different gaze patterns when simply viewing facial expressions, without being asked to actually identify the emotion. ${ }^{44}$ Decreased FA was found in adolescents with ASD bilaterally in the ILF, a pathway connecting the occipital cortex with the anterior temporal lobes and amygdala. FA values in the ILF were negatively correlated with ADOS social scores in both adolescents and adults. ${ }^{25}$ Concordant with these findings, low FA of ILF was related to poor social interaction in this study.

Another affected association tract was the SLF. In the left hemisphere, this fiber tract connects the Broca and Wernicke areas. Thus, abnormal connections between these two areas can cause communication problems, such as those observed in individuals with ASD. This finding is consistent with a fMRI study that found impaired functional connectivity between language areas in ASD using a sentence comprehension task. ${ }^{3}$ Moreover, several DTI studies also have revealed abnormalities of the left SLF in ASD. ${ }^{45-47}$ The importance of the right SLF in ASD is not less than that of the left SLF because of its connection to the superior temporal sulcus, ${ }^{48}$ a region known for its role in processing biologic motion, which is abnormal in ASD. ${ }^{49}$ Moreover, several DTI studies have revealed abnormalities of the right SLF. ${ }^{11,28,50,51}$ Finally, similar to the IFOF, the SLF also has a broad neuroanatomic range, connecting 
the frontal, parietal, and temporal lobes. This tract may also potentially serve an important role in linking all the components of brain structures involved in social cognition. In accordance with these findings, we found that impairment of SLF was related to poor communication, poor social interaction.

Our study must be interpreted in the context of several methodological limitations. The first is the small sample size. To compromise this limitation we used p-value less than 0.001 in statistical analyses of TBSS. The second is the wide range of age. In TBSS studies, age may have a significant effect on white matter differences. ${ }^{28}$ To resolve this factor, we regressed out the age factor in analyses. The third limitation is that this study only includes individuals with high-function autism. Nevertheless, we would anticipate that more severely affected individuals with ASD might have even more prominent disconnectivity between cortical areas. The forth, There are references $^{52-54}$ in the existing DTI studies that there is not a large difference in gender. Based on this, we thought that it would be more appropriate to include a woman in the covariate to control the gender effect to increase the statistical power due to the small sample size. However, this was not the case in most studies. So this point is clearly considered a limitation in this study. The final matter of consideration is the nature of the control group, which is composed of normally developing individuals without any delayed development independent of ASD. Therefore we cannot clearly demonstrate that these findings are specific to ASD. These limitations restrict generalize of the results to all ASD.

We found impairment of neural connectivity mainly in association fiber tracts in high-function autism as evidenced by decreased FA, an index of white matter integrity, of these tracts. Among them, IFOF, which connects the social brain, had significant relationships with various domains such as social interaction. The ILF and SLF also showed decreased FA in individuals with HFA. Decreased FA of ILF and SLF had negative correlations with poor social interaction. These findings indicate that widespread abnormalities in association fiber tracts may contribute to both core and associated symptoms of ASD. However, further research is needed to generalize these results to all ASD. Future research should be implemented with large samples, diverse range of age, patients with various degree of severity of the illness, and developmentally delayed controls without autism spectrum disorder.

\section{Acknowledgements}

This study was supported by a faculty research grant of Yonsei University College of Medicine (6-2008-0299). We would like to thank E-Worldediting (www.eworldediting.com) for English language editing (YS1602-14).

\section{REFERENCES}

1. American Psychiatric Association. Diagnostic and Statistical Manual of Mental Disorders, DSM-5. Washington, DC: American Psychiatric Association; 2013.

2. Castelli F, Frith C, Happe F, Frith U. Autism, Asperger syndrome and brain mechanisms for the attribution of mental states to animated shapes. Brain 2002;125:1839-1849.

3. Just MA, Cherkassky VL, Keller TA, Minshew NJ. Cortical activation and synchronization during sentence comprehension in high-functioning autism: evidence of underconnectivity. Brain 2004;127:1811-1821.

4. Kennedy DP, Redcay E, Courchesne E. Failing to deactivate: resting functional abnormalities in autism. Proc Natl Acad Sci U S A 2006; 103:8275-8280.

5. Villalobos ME, Mizuno A, Dahl BC, Kemmotsu N, Muller RA. Reduced functional connectivity between $\mathrm{V} 1$ and inferior frontal cortex associated with visuomotor performance in autism. Neuroimage 2005; 25:916-925.

6. Ameis SH, Fan J, Rockel C, Voineskos AN, Lobaugh NJ, Soorya L, et al. Impaired structural connectivity of socio-emotional circuits in autism spectrum disorders: a diffusion tensor imaging study. PLoS One 2011; 6:e28044.

7. Basser PJ, Pierpaoli C. Microstructural and physiological features of tissues elucidated by quantitative-diffusion-tensor MRI. J Magn Reson 1996;213:560-570.

8. Barnea-Goraly N, Kwon H, Menon V, Eliez S, Lotspeich L, Reiss AL. White matter structure in autism: preliminary evidence from diffusion tensor imaging. Biol Psychiatry 2004;55:323-326.

9. Lee JE, Bigler ED, Alexander AL, Lazar M, DuBray MB, Chung MK, et al. Diffusion tensor imaging of white matter in the superior temporal gyrus and temporal stem in autism. Neurosci Lett 2007;424:127132.

10. Alexander AL, Lee JE, Lazar M, Boudos R, DuBray MB, Oakes TR, et al. Diffusion tensor imaging of the corpus callosum in autism. Neuroimage 2007;34:61-73.

11. Cheung C, Chua SE, Cheung V, Khong PL, Tai KS, Wong TK, et al. White matter fractional anisotrophy differences and correlates of diagnostic symptoms in autism. J Child Psychol Psychiatry 2009;50:11021112.

12. Smith SM, Jenkison M, Johansen-Berg H, Rueckert D, Nichols TE, Mackay CE, et al. Tract-based spatial statistics: voxelwise analysis of multi-subject diffusion data. Neuroimage 2006;31:1487-1505.

13. Jones DK, Symms MR, Cercignani M, Howard RJ. The effect of filter size on VBM analyses of DT-MRI data. Neuroimage 2005;26:546-554.

14. Jou RJ, Mateljevic N, Kaiser MD, Sugrue DR, Volkmar FR, Pelphrey KA. Structural neural phenotype of autism: preliminary evidence from a diffusion tensor imaging study using tract-based spatial statistics. AJNR Am J Neuroradiol 2011;32:1607-1613.

15. Walker L, Gozzi M, Lenroot R, Thurm A, Behseta B, Swedo S, et al. Diffusion tensor imaging in young children with autism: biological effects and potential confounds. Biol Psychiatry 2012;15;72:1043-1051.

16. Wechsler D. The Wechsler Intelligence Scale for Children-fourth edition. London: Pearson Assessment; 2004.

17. Lord C, Risi S, Lambrecht L, Cook EH Jr, Leventhal BL, DiLavore PC, et al. The autism diagnostic observation schedule-generic: a standard measure of social and communication deficits associated with the spectrum of autism. J Autism Dev Disord 2000;30:205-223.

18. Lord C, Rutter M, Le Couteur A. Autism Diagnostic Interview-Revised: a revised version of a diagnostic interview for caregivers of individuals with possible pervasive developmental disorders. J Autism Dev Disord 1994;24;659-685.

19. Kim DJ, Park HJ, Kang KW, Shin YW, Kim JJ, Moon WJ, et al. How does distortion correction correlate with anisotropic indices? A diffusion tensor imaging study. Magn Reson Imaging 2006;4:1369-1376.

20. Anjari M, Srinivasan L, Allsop JM, Hajnal JV, Rutherford MA, Edward $\mathrm{AD}$, et al. Diffusion tensor imaging with tract-based spatial statistics reveals local white matter abnormalities in preterm infants. Neuroimage 2007;35:1021-1027. 
21. Nichols TE, Holmes AP. Nonparametric permutation tests for functional neuroimaging: a primer with examples. Hum Brain Mapp 2002; 15:1-25.

22. Smith SM, Nichols TE. Threshold-free cluster enhancement: addressing problems of smoothing, threshold dependence and localization in cluster inference. Neuroimage 2009;44:83-98.

23. Hua K, Zhang J, Wakana S, Jiang H, Li X, Reich DS, et al. Tract probability maps in stereotaxic spaces: analysis of white matter anatomy and tract-specific quantification. NeuroImage 2008;39:336-347.

24. Perkins TJ, Stokes MA, McGillivray JA, Mussap AJ, Cox IA, Maller JJ, et al. Increased left hemisphere impairment in high-functioning autism: a tract based spatial statistics study. Psychiatry Res 2014;224:119-123.

25. Bakhtiari R, Zürcher NR, Rogier O, Russo B, Hippolyte L, Granziera C, et al. Differences in white matter reflect atypical developmental trajectory in autism: A Tract-based Spatial Statistics study. NeuroImage Clin 2012;1:48-56.

26. Jou RJ, Jackowski AP, Papademetris X, Rajeevan N, Staib LH, Volkmar FR. Diffusion tensor imaging in autism spectrum disorders: preliminary evidence of abnormal neural connectivity. Aust N Z J Psychiatry 2011;45:153-162.

27. Shukla DK, Keehn B, Muller RA. Tract-specific analyses of diffusion tensor imaging show widespread white matter compromise in autism spectrum disorder. J Child Psychol Psychiatry 2011;52:286-295.

28. Catani M, Jones DK, Donato R, Ffytche DH. Occipito-temporal connections in the human brain. Brain 2003;126:2093-2107.

29. Martino J, Brogna C, Robles SG, Vergani F, Duffau H. Anatomic dissection of the inferior fronto-occipital fasciculus revisited in the lights of brain stimulation data. Cortex 2010;46:691-699.

30. Thomas C, Avidan G, Humphreys K, Jung K, Gao F, Behrmann M. Reduced structural connectivity in ventral visual cortex in congenital prosopagnosia. Nat Neurosci 2009;12:29-31.

31. Rizzolatti G, Matelli M. Two different streams from the dorsal visual system. Exp Brain Res 2003;153:146-157.

32. Catani M, Jones DK, Donato R, Ffytche DH, Occipito-temporal connections in the human brain. Brain 2003;126:2093-2107.

33. Wakana S, Jiang H, Nagae-Poetscher LM, van Zijl PC, Mori S. Fiber tract based atlas of human white matter anatomy. Radiology 2004;230: 77-87.

34. Thiebaut de Schotten M, Dell'Acqua F, Valabregue R, Catani M. Monkey to human comparative anatomy of the frontal lobe association tracts. Cortex 2012;48:82-96.

35. Philippi CL, Mehta S, Grabowski T, Adolphs R, Rudrauf D. Damage to association fiber tracts impairs recognition of the facial expression of emotion. J Neurosci 2009;29:15089-15099.

36. Boucher J. Putting theory of mind in its place: psychological explanations of the socio-emotional-communicative impairments in autistic spectrum disorder. Autism 2012;16:226-246.

37. Losh M, Adolphs R, Poe MD, Couture S, Penn D, Baranek GT, et al. Neuropsychological profile of autism and the broad autism phenotype. Arch Gen Psychiatry 2009;66:518-526.

38. Weng SJ, Carrasco M, Swartz JR, Wiggins JL, Kurapati N, Liberzon I, et al. Neural activation to emotional faces in adolescents with autism spectrum disorders. J Child Psychol Psychiatry 2011;52:296-305.

39. Pelphrey KA, Carter EJ. Charting the typical and atypical development of the social brain. Dev Psychopathol 2008;20:1081-1102.

40. Adolphs R, Tranel D. Intact recognition of emotional prosody following amygdala damage. Neuropsychologia 1999;37:1285-1292.

41. Celani G, Battacchi MW, Arcidiacono L. The understanding of the emotional meaning of facial expressions in people with autism. J Autism Dev Disord 1999;29:57-66.

42. Gross TF. The perception of four basic emotions in human and nonhuman faces by children with autism and other developmental disabilities. J Abnorm Child Psychol 2004;32:469-480.

43. Harms MB, Martin A, Wallace GL. Facial emotion recognition in autism spectrum disorders: a review of behavioral and neuroimaging studies. Neuropsychol Rev 2010;20:290-322.

44. Klin A, Jones W, Schultz R, Volkmar, Cohen D. Visual fixation patterns during viewing of naturalistic social situations as predictors of social competence in individuals with autism. Arch Gen Psychiatry 2002;59: 809-816.

45. Barnea-Goraly N, Lotspeich LJ, Reiss AL. Similar white matter aberrations in children with autism and their unaffected siblings: a diffusion tensor imaging study using tract-based spatial statistics. Arch Gen Psychiatry 2010;67:1052-1060.

46. Sahyoun CP, Belliveau JW, Mody M. White matter integrity and pictorial reasoning in high-functioning children with autism. Brain Cogn 2010;73:180-188.

47. Fletcher PT, Whitaker RT, Tao R, DuBray MB, Froehlich A, Ravichandran $\mathrm{C}$, et al. Microstructural connectivity of the arcuate fasciculus in adolescents with high-functioning autism. Neuroimage 2010;51:1117-1125.

48. Catani M, Jones DK, Ffytche DH. Perisylvian language networks of the human brain. Ann Neurol 2005;57:8-16.

49. Kaiser MD, Hudac CM, Shultz S, Lee SM, Cheung C, Berken AM, et al. Neural signatures of autism. Proc Natl Acad Sci U S A 2010;107:2122321228.

50. Kumar A, Sundaram SK, Sivaswamy L, Behen ME, Makki MI, Ager J, et al. Alterations in frontal lobe tracts and corpus callosum in young children with autism spectrum disorder. Cereb Cortex 2010;20:2103-2113.

51. Cheng Y, Chou KH, Chen IY, Fan YT, Decety J, Lin CP. Atypical development of white matter microstructure in adolescents with autism spectrum disorders. Neuroimage 2010;50:873-882.

52. Assis ZA, Bagepally BS, Saini J, Srinath S, Bharath RD, Naidu PR, et al. Childhood autism in India: a case-control study using tract-based spatial statistics analysis. Indian J Psychiatry 2015;57:272-277.

53. Billeci L, Calderoni S, Tosetti M, Catani M, Muratori F. White matter connectivity in children with autism spectrum disorders: a tract-based spatial statistics study. BMC Neurol 2012;12:148.

54. Bradstreet LE, Hecht EE, King TZ, Turner JL, Robins DL. Associations between autistic traits and fractional anisotropy values in white matter tracts in a nonclinical sample of young adults. Exp Brain Res 2017;235: 259-267. 